\title{
A COMPARISON OF PROPHYLACTIC, TOPICAL AND SUBCONJUNCTIVAL TREATMENT IN CATARACT SURGERY
}

\author{
ROSHINI SANDERS, CAROLINE J. MACEWEN, W. M. HAINING \\ Dundee
}

\begin{abstract}
SUMMARY
Prophylactic subconjunctival antibiotics and steroids have been used in cataract surgery since the early 1950s. However despite their widespread acceptance, there has, to date, been no direct comparison of subconjunctival and topical routes of administration.

We therefore carried out a prospective comparison of the effects of subconjunctival and topical administration of antibiotics and steroids in sixty patients undergoing elective, uncomplicated, extracapsular cataract extraction and posterior chamber lens implantation. The patients were examined by a single observer on the first and third post-operative days and two and six weeks following surgery. The observer was blinded to the patient treatment group.

Significantly higher degrees of conjunctival injection and anterior chamber activity were noted in those who received subconjunctival injections compared with those who received topical treatment. There were no significant differences between the two groups in best corrected visual acuity, central corneal oedema, cystoid macular oedema, intraocular pressure and infection.

We conclude that prophylactic subconjunctival therapy in uncomplicated cataract surgery is not necessary.

The use of prophylactic antibiotics in intraocular surgery was introduced in the late 1940s, when Sorsby and Ungar described the first subconjunctival injection of Penicillin ${ }^{1}$. They experimentally proved that this route achieved higher and more persistent intraocular concentrations of antiobiotic than topically or systemically.

Over the next few decades the use of prophylactic subconjunctival therapy in cataract surgery became well established. A study done in 1956 on 5,000 cases recorded a post-operative infection rate of zero when subconjunctival antibiotics were used as opposed to $9 \%$ when topical antiobiotic drops were substituted ${ }^{2}$.

A further study in 1971 with 2,000 cases demonstrated a significant decrease in post-operative complications

Department of Ophthalmology, Ninewells Hospital \& Medical School, Dundee.

Correspondence to: Dr R. Sanders, Department of Ophthalmology, Ninewells Hospital \& Medical School, Dundee DD1 9SY.
\end{abstract}

such as uveitis, shallowing of the anterior chamber, iris prolapse, wound dehiscence and secondary glaucoma when subconjunctival steroids were used ${ }^{3}$.

Earliest doubts were cast on the exact indication for the subconjunctival route in the mid 1970s, when it was demonstrated that subconjunctival steroids were less effective in suppressing corneal inflammation than topical drops of steroid ${ }^{4}$. Evidence was presented which showed that increased steroid levels in the cornea equated with increased anti-inflammatory effect, but only up to a point. Once this point had been exceeded the excess steroid caused corneal toxicity with more subsequent ocular inflammation, pain and greater systemic absorption.

In addition it was suggested that the use of subconjunctival antibiotics led to a delayed and more protracted post-operative infection with a poorer visual outcome in the long term ${ }^{5}$. A further extensive study of 10,000 cases indicated no reduction in immediate post-operative endopthalmitis when subconjunctival antibiotics were used as opposed to topical antibiotics ${ }^{6}$.

In the last decade there have been no clear guidelines for the use of prophylactic subconjunctival antibiotics and steroids in cataract surgery. We therefore set up a prospective study to evaluate the effect of prophylactic subconjunctival therapy, as compared with topical drops in cataract surgery. Particular attention was paid to the degree of post-operative inflammation and infection occurring with the two different routes of treatment.

\section{PATIENTS AND METHODS}

The study group consisted of sixty consecutive patients who underwent elective, uncomplicated cataract surgery. Any patient who was diabetic, had suffered from previous eye disease or was on oral steroids or anti-inflammatory drugs was excluded from the study. All patients had senile or pre-senile cataracts with no known pre-disposing factor. The patients were randomised to receive a subconjunctival injection or topical drops post-operatively. Pre-operatively all patients received g. Chloramphenicol q.i.d. to both eyes for twenty-four hours. The operation was performed by one of two experienced surgeons. 
Table I. Grades of AC Activity. Grading of AC Activity in patients following cataract surgery. Each grade is given a score.

\begin{tabular}{lc}
\hline AC Activity (Flare \& Cells) & Score \\
\hline 0 (Absent) & 0 \\
1 (Mild) & 1 \\
2 (Moderate) & 2 \\
3 (Severe) & 3 \\
4 (Fibrin) & 4 \\
\hline
\end{tabular}

Table II. Grades of AC Conjunctival Injection. Grading of conjunctival injection in patients following cataract surgery. Each grade is given a score.

\begin{tabular}{lc}
\hline Conjunctival Injection & Score \\
\hline 0 (Absent) & 0 \\
1 (Mild) & 1 \\
2 (Moderate) & 2 \\
3 (Severe) & 3 \\
\hline
\end{tabular}

Table III. Visual Acuity Six Weeks Post-Operatively. Best corrected visual acuity six weeks following cataract surgery, in all patients in Group D and Group S.

\begin{tabular}{lcccccccc}
\hline & $6 / 6$ & $6 / 9$ & $6 / 12$ & $6 / 18$ & $6 / 24$ & $6 / 36$ & $6 / 60$ & $\mathrm{CF}$ \\
\hline $\begin{array}{l}\text { Group D } \\
\text { (Drops) }\end{array}$ & 8 & 10 & 6 & 1 & 1 & 1 & 2 & 1 \\
$\begin{array}{l}\text { Group S } \\
\text { (Subconj) }\end{array}$ & 5 & 10 & 9 & 3 & 0 & 1 & 1 & 1 \\
\hline
\end{tabular}

Table IV. Frequency of Grades of Conjunctival Injection PostOperatively. The frequency with which each grade of conjunctival injection was noted in patients in Group D and Group S, at each postoperative examination.

\begin{tabular}{lrrrrrrrrr}
\hline & \multicolumn{2}{c}{ Day 1 } & \multicolumn{2}{c}{ Day 3 } & \multicolumn{2}{c}{ 2 Weeks } & \multicolumn{2}{c}{ 2 Months } \\
\hline Grades & D & S & \multicolumn{1}{c}{ D } & S & D & S & D & S \\
0 & 0 & 0 & 3 & 1 & 9 & 9 & 29 & 26 \\
1 & 17 & 10 & 18 & 11 & 17 & 14 & 1 & 1 \\
2 & 12 & 19 & 8 & 17 & 4 & 7 & 0 & 3 \\
3 & 1 & 1 & 1 & 1 & 0 & 0 & 0 & 0 \\
\hline
\end{tabular}

$\mathrm{D}=$ Drops; $\mathrm{S}=$ Subconj.

Twenty eight patients had a general anaesthetic and thirty two had local retrobulbar anaesthesia.

The surgical technique was standardised and all patients underwent a limbal section followed by endocapsular cataract extraction. One piece PMMA 720A intraocular implants were used in all cases. The cataract section was closed with $10^{\prime} 0$ nylon sutures and the conjunctiva with $10^{\prime} 0$ virgin silk sutures. At the end of surgery one group had a subconjunctival injection of betnesol $(2 \mathrm{mg})$ and gentamicin $(20 \mathrm{mg})$ (Group S), and the other a drop of betnesol $(0.1 \%)$ /neomycin $(0.5 \%)$ (Group D). The post-operative regime consisted of g. betnesol $\mathrm{N}$ q.i.d. and g. cyclopentolate $1 \%$ b.d. for two weeks and b.d. and o.d. respectively in the third to sixth weeks following surgery. Patients were discharged home on or following the third post-operative day.

All patients were examined by a single observer, blinded to the patient treatment group, on the first and third post-operative day and two and six weeks following surgery. Post-operative inflammation and infection was assessed by recording the degree of anterior chamber activity and conjunctival injection, the presence of central corneal oedema and clinical evidence of external or intraocular infection. Additional parameters recorded were visual acuity, intraocular pressure and the presence of cystoid macular oedema.

Anterior chamber activity was graded using a modification of the method described by Hogan into Grades $0-4^{7}$. A standard slit lamp beam of $1 \mathrm{~mm}$ width and $3 \mathrm{~mm}$ length set at maximal light intensity and magnification was used to assess the presence of cells and fibrin in the anterior chamber. Special attention was paid to documenting the presence of inflammatory cells and excluding pigmented or red blood cells. Grade O represented a completely quiet anterior chamber. Grade 1 represented mild (approx. up to 10 cells per field) and Grade 2 moderate (10-20 cells) AC activity. Grade 3 represented severe AC activity (approx. $>20$ cells). The presence of fibrin, keratic precipitates or hypopyon was given a grading of 4 . Each grade was given a score of $0-4$ (Table I).

Conjunctival injection was graded in a similar fashion. A completely white eye was given a grading of 0 . Grade 1 represented the mildest of injection such as a few congested conjunctival vessels in one or two quadrants of the eye. Grade 2 represented a more diffuse conjunctival injection with involvement of the deeper episcleral vessels and

Table V. Post-Operative Conjunctival Injection Scores. The statistical analysis of the mean score difference in conjunctival injection postoperatively between Groups S and D.

\begin{tabular}{lcccc}
\hline Post-Op Visit & Day 1 & Day 3 & 2 Weeks & 2 Months \\
\hline Mean Score & & & & \\
Difference & & & & \\
Between Groups & & & & \\
S \& D & 0.23 & 0.37 & 0.10 & 0.20 \\
Standard Error & 0.14 & 0.17 & 0.18 & 0.12 \\
95\% Confidence & & & & \\
Interval & $-0.05,0.51$ & $0.03,0.71$ & $-0.26,0.46$ & $-0.04,0.44$ \\
\hline
\end{tabular}

Table VI. Frequency of Grades of AC Activity Post-Operatively. The frequency with which each grade of AC Activity was noted in patients in Group D and Group S at each post-operative examination.

\begin{tabular}{lrrrrrrrr}
\hline & \multicolumn{2}{c}{ Day 1} & \multicolumn{2}{c}{ Day 3} & \multicolumn{2}{c}{ 2 Weeks } & \multicolumn{2}{c}{2 Months } \\
\hline Grades & D & S & D & \multicolumn{1}{c}{ S } & D & S & D & S \\
0 & 0 & 0 & 0 & 0 & 6 & 7 & 24 & 22 \\
1 & 6 & 0 & 14 & 5 & 20 & 17 & 6 & 6 \\
2 & 22 & 24 & 15 & 21 & 4 & 5 & 0 & 2 \\
3 & 2 & 6 & 1 & 4 & 0 & 0 & 0 & 0 \\
4 & 0 & 0 & 0 & 0 & 0 & 1 & 0 & 0 \\
\hline
\end{tabular}

$\mathrm{D}=$ Drops; $\mathrm{S}=$ Subconj.

Table VII. Post-Operative AC Activity Scores. The statistical analysis of the mean score difference in Ac activity post-operatively between Groups S and D.

\begin{tabular}{lcccc}
\hline Post-Op Visit & Day 1 & Day 3 & 2 Weeks & 2 Months \\
\hline Mean Score & & & & \\
Difference & & & & \\
Between Groups & & & & \\
S \& D & 0.33 & 0.40 & 0.10 & 0.10 \\
Standard Error & 0.12 & 0.15 & 0.19 & 0.13 \\
95\% Confidence & & & & \\
Interval & $0.09,0.57$ & $0.1,0.7$ & $-0.28,0.48$ & $-0.16,0.36$ \\
\hline
\end{tabular}




\section{BAR CHART A \\ Comparison of Conjunctival Injection Grades Post-Operatively in Groups S and D}

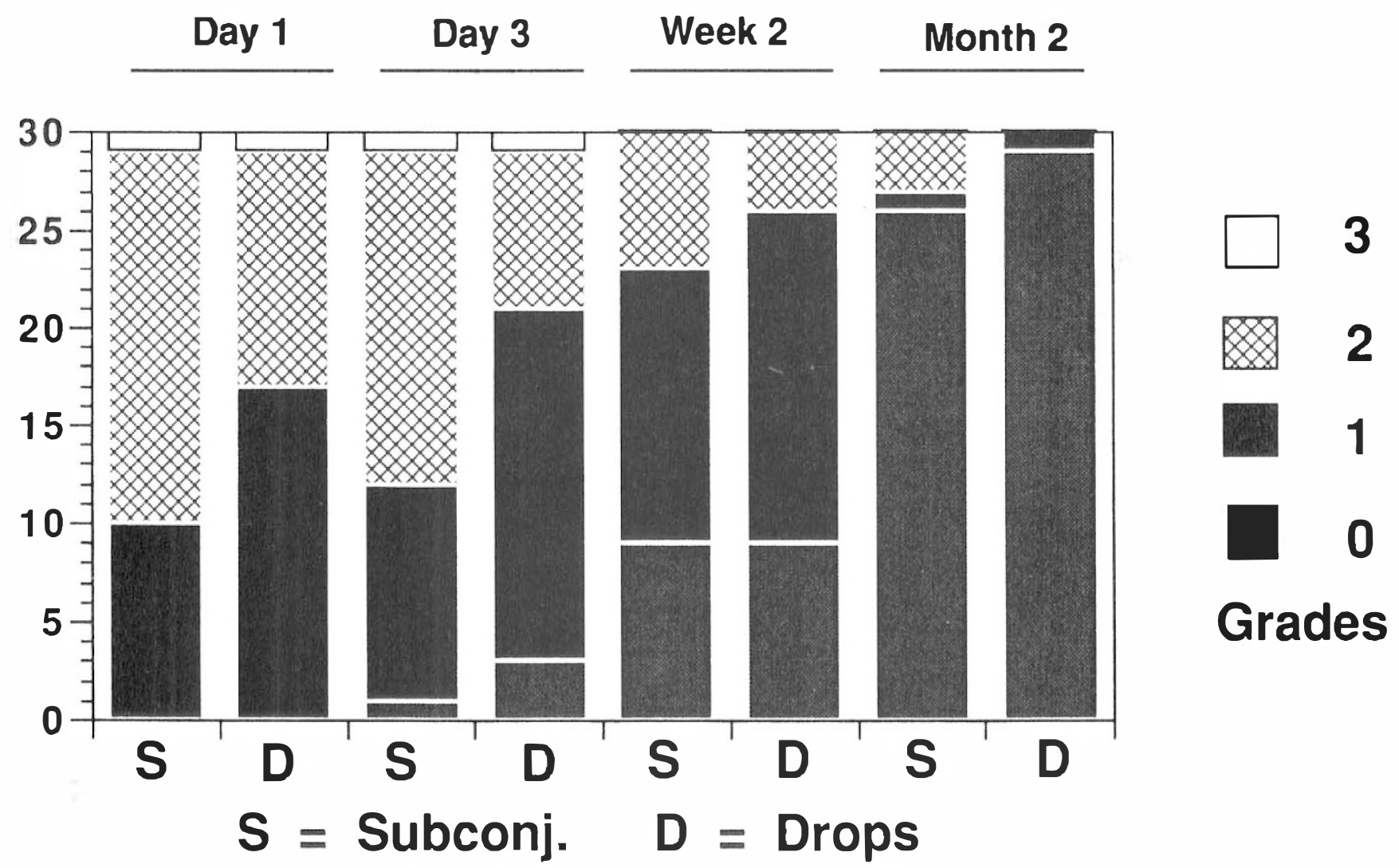

Bar Chart A. Comparison of Conjunctival Injection Grades Post-Operatively in Groups $S$ and D. Bar chart showing a comparison between Groups $S$ and D, of grades of conjunctival injection seen at each post-operative examination.

absence or presence of a small subconjunctival haemorrhage. The presence of intense diffuse conjunctival injection, conjunctival chemosis or massive subconjunctival

Graph I COMPARISON OF CONJUNCTIVAL INJECTION SCORES Post-operatively in groups $S$ and $D$

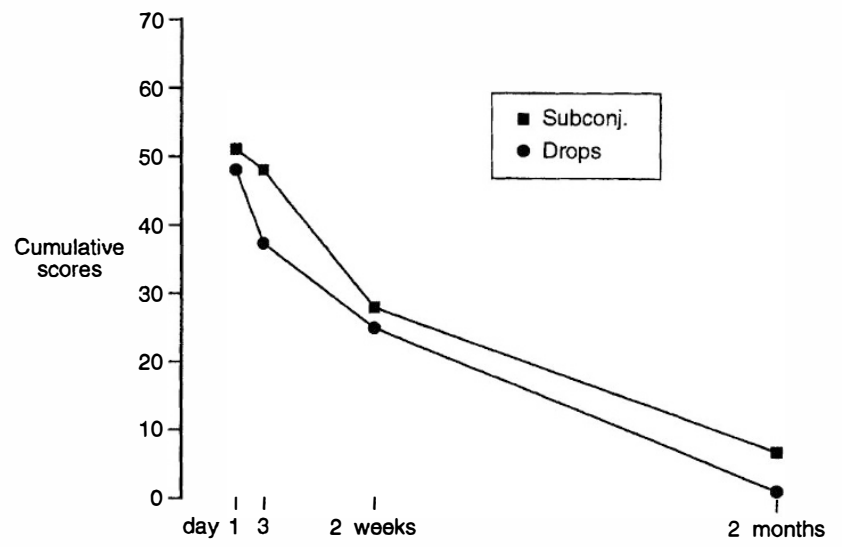

Graph 1. Comparison of Conjunctival Injection Scores PostOperatively in Groups $S$ and D. Graph showing the additive score of conjunctival injection in all patients in Groups $S$ and $D$ at each post-operative examination. haemorrhage was given a grading of 3. Each grade was given a score of 0-3 (Table II).

Cystoid macular oedema was diagnosed by sterescopic biomicroscopy.

\section{RESULTS}

The study group consisted of twenty-four males and thirty-six females. Patients ages ranged from 45-92 years in Group $S$ with an average age of 74.6 years in Group D the age range was from 51 to 91 years with an average age of 76.4 years.

Table III charts best corrected visual acuity in each group six weeks following surgery. Forty-eight patients achieved visual acuities of 6/6-6/12 and 12 patients visual acuities of 6/18-C.F. Poor vision in the latter group was due to central corneal oedema, cystoid macular oedema and senile macular degeneration. Six months following surgery visual acuity improved to $6 / 18$ or better in six patients due to resolution of central corneal oedema or cystoid macular oedema. Using a chi-squared test for trend no significant difference in visual acuity was found between the two groups $(\mathrm{p}=0.46)$.

Table IV shows the frequency with which the different 


\section{BAR CHART B}

\section{Comparison of AC Activity Grades Post-Operatively in Groups S \& D.}

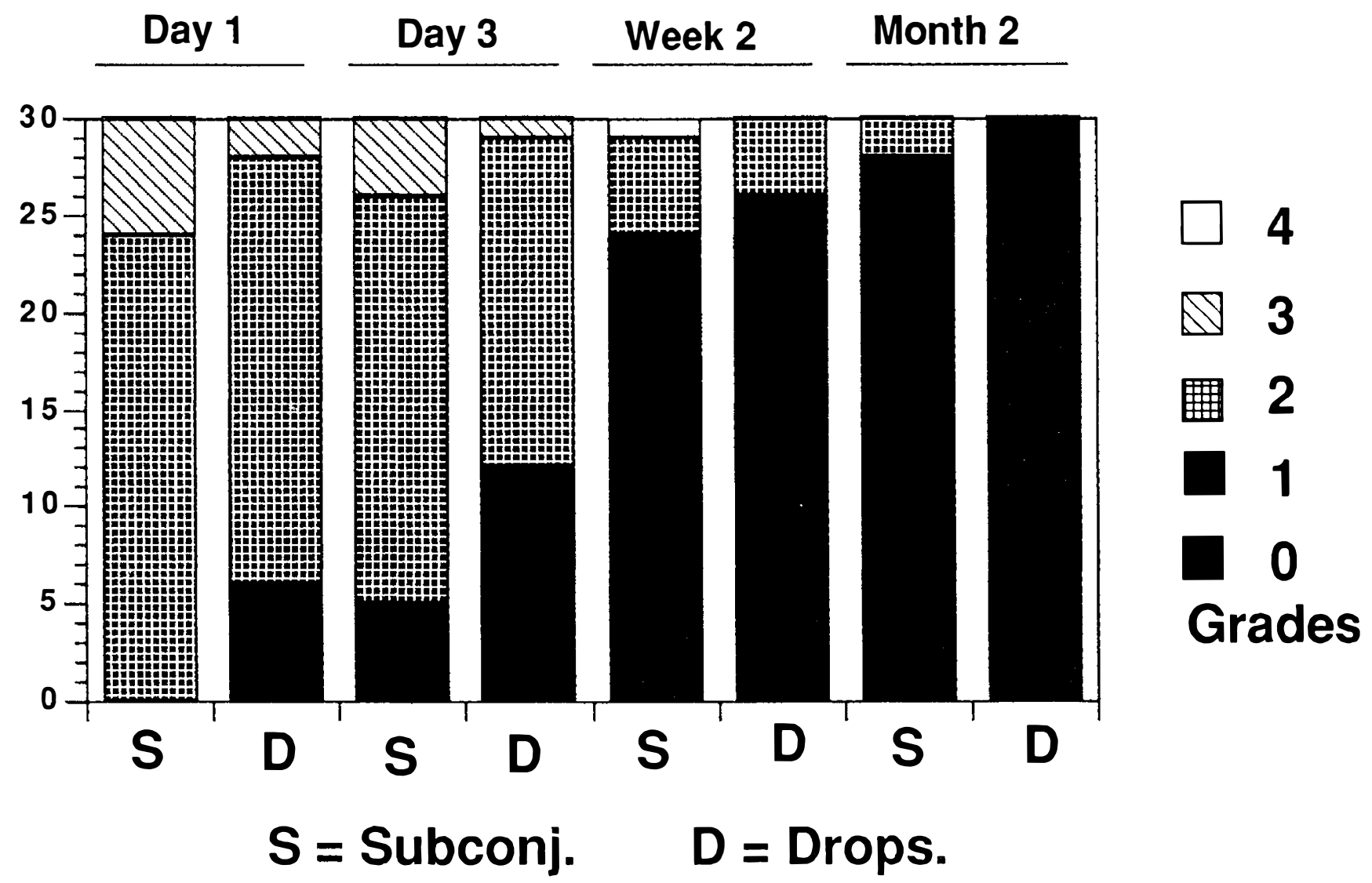

Bar Chart B. Comparison of AC Activity Grades Post-Operatively in Groups S and D. Bar chart showing a comparison between Groups $S$ and $D$ of grades of AC activity seen at each post-operative examination.

grades of conjunctival injection were noted in the two groups at each post-operative examination. On the first post-operative day the majority of patients who had received a subconjunctival injection (Group S) had Grade 2 conjunctival injection, while the majority of patients that had drops had Grade 1 injection. Two months after surgery four patients in Group S still had Grade 1 or 2 conjunctival injection while only one patient in Group D had Grade 1 injection. The remaining 55 patients had complete resolution of conjunctival injection. Bar Chart A shows increasing resolution of conjunctival injection in both groups with each examination. Using the scoring system, Graph 1 illustrates the additive scores of all patients in each group at each examination. Group $\mathrm{S}$ achieved higher scores and therefore a greater degree of conjunctival injection at each examination than Group D. This difference is more apparent in the early post-operative period.

Table V shows the mean score difference between each group at each post-operative visit. A significantly greater degree of conjunctival injection $(p<0.05)$ was seen in Group $S$ than Group D on the third post-operative day.
This was still found when analysis of variance was done to adjust for effects of type of anaesthetic.

Anterior chamber activity has been tabulated and graphed likewise (Table VI, Bar Chart B). Again the trend in each group with each examination is towards resolution. On the first post-operative day the majority of patients in each group had Grade 2 AC activity. There were however six patients in Group S who had Grade 3 AC activity while only two patients in Group D had Grade 3 AC activity. Only one patient developed a fibrinous uveitis (Grade 4 AC activity) two weeks following surgery and this patient had received subconjunctival therapy. Two months after surgery eight patients in Group S had Grade 1 or 2 AC activity and six patients in Group D had Grade 1 AC activity. The remaining forty-six patients had complete resolution of AC activity. Graph 2 shows that Group $\mathrm{S}$ achieved higher scores than Group D at each visit, implying a greater degree of anterior chamber activity. Again this difference is more apparent in the early postoperative period.

Table VII shows the mean score different between each 
Graph ॥ COMPARISON OF AC ACTIVITY SCORES

Post-operatively in groups $S$ and $D$

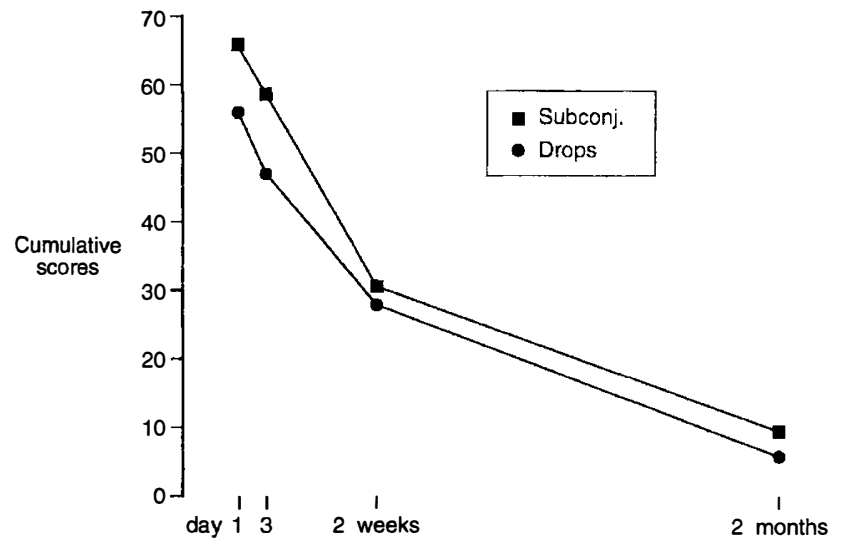

Graph 2. Comparison of AC Activity Scores Post-Operatively in Groups $S$ and D. Graph showing the additive score of $A C$ activity in all patients in Groups $S$ and $D$ at each post-operative examination.

group at each post-operative visit. A significantly greater degree of AC activity $(\mathrm{p}<0.05)$ was seen in Group $S$ than Group D on the first and third post-operative day. This was still found when analysis of variance was done to adjust for effects of type of anaesthetic.

Central corneal oedema was observed in five patients in Group S and three patients in Group D on the first postoperative day. Three cases persisted six weeks after surgery in Group $\mathrm{S}$ with complete resolution of those in Group D. This was not statistically significant.

All recorded intraocular pressures were less than $23 \mathrm{mmHg}$ with the exception of one patient who developed a pressure of $60 \mathrm{mmHg}$ on the first post-operative day. This was due to the inadvertent failure to aspirate Sodium Hyaluronate at the end of surgery.

Cystoid Macular Oedema was noted in three patients in Group D and one patient in Group S six weeks postoperatively. Six months following surgery two cases persisted in Group D with complete resolution of those in Group S. This was not statistically significant.

There were no cases of conjunctival, corneal or intraocular infection post-operatively in either group.

\section{DISCUSSION}

Techniques of cataract surgery have changed greatly in the last forty years. The most significant advances have been the introduction of the operating microscope in association with finer instruments, suturing material and improved surgical methods. Taken together with improved sterilisation methods, these have reduced the degree of inflammation and infection occuring post-operatively. Prophylactic subconjunctival therapy in cataract surgery was established decades ago before these advances in surgery. We therefore felt that the subconjunctival and topical routes should be compared in a series of consecutive patients undergoing cataract surgery.
The two parameters that initially showed statistically significant differences between the two groups were the degree of conjucntival injection and anterior chamber activity. Group S (sub-conjunctival injection) had a significantly greater degree of conjunctival injection than Group D (Drops). Factors contributing towards this are possibly the local physical trauma to the conjunctiva of having a volume of fluid injected subconjunctival as well as local antibiotic toxicity. Recent reports in the literature describe the toxicity of subconjunctival gentamicin leading to closure and leakage of conjunctival and episcleral vessels causing significant conjunctival chemosis, injection and pain ${ }^{8}$.

Group S also had a significantly greater degree of anterior chamber activity than Group D. Again the trauma induced by a subconjunctival injection may be a causative factor. The volume of fluid introduced subconjunctivally alternatively may cause a rise in pressure leading to an element of anterior segment ischaemia.

The high dose of steroids and antibiotics in the eye following subconjunctival injection is principally due to absorption across the cornea ${ }^{9,10}$. This may be in excess of therapeutic requirements leading to corneal toxicity and oedema. The corneal toxicity may lead to secondary anterior chamber activity.

No statistical difference was found in best corrected visual acuity between the two groups. Central corneal oedema was seen more frequently in Group $S$ and cystoid macular oedema in Group D. These findings however did not achieve statistical significance. The correlation between cystoid macular oedema and the degree of anterior chamber activity has already been demonstrated $^{11}$. All cases of cystoid macular oedema in our study had Grade 2 anterior chamber activity.

There were no cases of post-operative infection in our study. However modern rates of post-operative infection are extremely low and therefore our series is not large enough to draw any conclusions about this complication. We therefore looked retrospectively at all cases of immediate post-operative endophthalmitis in our department following cataract surgery between the years 19851990. Our department performs over 1,500 cases of cataract surgery annually. There has been a gradual change from subconjunctival to topical prophylactic therapy during this five year period. Six patients developed endophthalmitis during these years. Five patients had received a subconjunctival injection of betnesol or betnesol and gentamicin and one had received a drop of betnesol $\mathrm{N}$.

Our study shows that the routine use of combined prophylactic subconjunctival steroids and antibiotics in uncomplicated cataract surgery in otherwise healthy eyes is unnecessary, and may in fact increase the degree of postoperative uveitis and conjunctival reaction. Our study numbers however are not large enough to draw any conclusions regarding the effect on the rate of post-operative infection. Other large studies however have indicated that prophylactic subconjunctival antibiotics do not alter the risk of post-operative infection ${ }^{5.6}$. 
We would like to thank Mrs Lynda Rose for typing the manuscript, and $\mathrm{Mr} \mathrm{S}$. Ogston and Mr C. Guthrie for help with the statistics.

Key words: Antibiotics, Cataract Surgery, Steroids, Subconjunctival, Topical.

\section{REFERENCES}

1. Sorsby A, Ungar J: Distribution of Penicillin in the Eye after Subconjunctival Injection. $\mathrm{Br} J$ Ophthalmol 1947, 31: 517-28.

2. Pearlman MD: Prophylactic Subconjunctival Penicillin and Streptomycin after Cataract Extraction. Arch Ophthalmol 1956, 55: 516-18.

3. Buxton JN, Smith DE, Brownstein S: Cataract Extraction and Subconjunctival Repository Corticosteroids. Ann Ophthalmol 1971, 3: 1376-9.

4. Leibowitz HM and Kupferman A. Periocular Injection of Corticosteroids. Arch Ophthalmol 1977, 95: 311-14.
5. Aronsfam RH: Pitfalls of Prophylaxis. Am J ophthalmol 1964, 57: 312-15.

6. Christy NE and Lall P: Postoperative Endopthalmitis Following Cataract Surgery. Arch Ophthalmol 1973, 90: 351-66.

7. Hogan MH, Kimura SJ, Thygeson P: Signs and Symptoms of Uveitis. Am J Ophthalmol 1959, 47: 155-70.

8. Jenkins CDG, McDonnell PJ, Spalton DJ. Randomised Single Blind Trial to Compare the Toxicity of Subconjunctival Gentamicin and Cefursxime in Cataract Surgery. Br J Ophthalmol 1990, 74: 734-8.

9. Clements DB and Tailor R: A Study of Aqueous and Serum Levels of Ceftanidine Following Subconjunctival Injection. Br J Ophthalmol 1987, 71: 433-5.

10. Tsiji A, Tamai I, Sasaki K. Intraocular Penetration Kinetics of Prednisolone after Subconjunctival Injection in Rabbits. Ophthalmol Research 1988, 20: 31-43.

11. Hitchings RA, Chisholm IH, Bird AC. Aphakic Cystoid Macular Oedema: Incidence and Pathogenesis. Invest Ophthalmol 1975, 14: 68-72. 